\title{
Black rings in more than five dimensions
}

\author{
Burkhard Kleihaus, Jutta Kunz and Eugen Radu \\ Institut für Physik, Universität Oldenburg, D-26111 Oldenburg, Germany
}

June 13, 2018

\begin{abstract}
We construct balanced black ring solutions in $d \geq 6$ spacetime dimensions, by solving the Einstein field equations numerically with suitable boundary conditions. The black ring solutions have a regular event horizon with $S^{1} \times S^{d-3}$ topology, and they approach the Minkowski background asymptotically. We analyze their global and horizon properties. The Smarr formula is well satisfied.
\end{abstract}

Introduction.- In 4 dimensions the stationary, asymptotically flat vacuum black holes (BHs) are given by the Kerr family. A spatial section of their event horizon has the topology of a two-sphere $S^{2}$. The Kerr BHs are uniquely characterized by their mass and angular momentum; thus these two numbers suffice to completely specify a vacuum BH space-time.

The generalizations of the Kerr BHs to $d \geq 5$ dimensions were found by Myers and Perry (MP) [1. Their global charges are the mass and $N=[(d-1) / 2]$ independent angular momenta. Their horizon topology is that of a $(d-2)$-sphere $S^{d-2}$. However, presenting heuristic arguments, Myers and Perry argued that in higher dimensions also black rings (BRs) might exist, and thus black objects with a different horizon topology.

In 2001 Emparan and Reall found such BR solutions in 5 dimensions. These are asymptotically flat and possess a horizon topology $S^{2} \times S^{1}$ [2]. Considering singly rotating BRs, Emparan and Reall showed that, for fixed mass, there are two branches of balanced black rings, a branch of thin black rings and a branch of fat black rings. These two branches merge at a minimal value of the angular momentum $j$, where their horizon area $a_{H}$ exhibits a cusp. This minimal value of $j$ of the BRs is smaller than the maximal value of $j$ of the MP BHs. Thus, within the range $j_{\min }^{B R}<j<j_{\max }^{M P}$, for given global charges 3 distinct solutions exist. Clearly, uniqueness is violated for these 5 -dimensional stationary vacuum solutions.

The discovery of the BRs spurred a lot of interest in BH solutions in higher dimensions. With many more solutions found, such as, in particular, composite objects of BHs and BRs, an intriguing phase diagram of vacuum black objects in 5 dimensions emerged (see e.g. [3, 4] for reviews of these aspects). In more than 5 dimensions, however, exact solutions of BRs or composite black objects could not yet be obtained, since no general analytic framework seems to exist for the construction of black objects with nonspherical horizon topology in $d>5$. 
A heuristic way to construct BRs is to bend a Schwarzschild black string and then achieve balance by spinning it along the $S^{1}$ direction [2]. This may be considered as the underlying picture for approximate techniques, such as the method of matched asymptotic expansion [5, 6. Here the central assumption is that some black objects, in certain ultra-spinning regimes, may be approximated by thin black strings or branes, curved into a given shape and boosted appropriately.

For BRs in $d>5$ dimensions this approach has led to approximate solutions, valid for configurations with a sufficiently large radius of the ring [5]. However, this approach cannot capture features that are expected to occur at moderate values of the angular momentum, where the radius of the $S^{1}$ of the ring is no longer large as compared to the radius of the $S^{d-3}$-sphere. Thus, in particular, it cannot deal with the conjectured transition of BRs to BHs with spherical horizon topology [5], i.e., the transition to the branch of pinched black holes emerging from a point of instability of the MP BHs [7, 8].

In this work we propose a nonperturbative approach to the construction of $d>5$ vacuum BRs. Here we obtain these solutions by solving numerically the Einstein equations with suitable boundary conditions. The numerical results for $d=6$ allow us to confirm the thin BR part of the phase diagram proposed in 5] and, in addition, to find a branch of fat BRs, which extends towards a horizon topology changing solution.

A new coordinate system.- A main difficulty in the construction of BR solutions is to find an appropriate coordinate system. The numerical solutions in this work are found for a parametrization of $D$-dimensional flat space

$$
d s_{D}^{2}=V_{1}\left(d r^{2}+r^{2} d \theta^{2}\right)+V_{2} d \Omega_{D-3}^{2}+V_{3} d \psi^{2}
$$

where $V_{1}=\frac{1}{U}, \quad V_{2}=r^{2}\left(\cos ^{2} \theta-\frac{1}{2}\left(1+\frac{R^{2}}{r^{2}}-U\right)\right), \quad V_{3}=r^{2}\left(\sin ^{2} \theta-\frac{1}{2}\left(1-\frac{R^{2}}{r^{2}}-U\right)\right)$, with $U=\sqrt{1+\frac{R^{4}}{r^{4}}-\frac{2 R^{2}}{r^{2}} \cos 2 \theta}$. The coordinate range in (1) is $0 \leq r<\infty, 0 \leq \theta \leq \pi / 2,0 \leq$ $\psi \leq 2 \pi, d \Omega_{D-3}^{2}$ is the metric on the unit sphere $S^{D-3}$, and $R>0$ is an arbitrary parameter. The coordinate transformation $\rho=r \sqrt{U}, \tan \Theta=\left(\frac{r^{2}+\rho^{2}+R^{2}}{r^{2}+\rho^{2}-R^{2}}\right) \tan \theta$, leads to a more usual parametrization of the $D>3$ flat space, $d s^{2}=d \rho^{2}+\rho^{2}\left(d \Theta^{2}+\cos ^{2} \Theta d \Omega_{D-3}^{2}+\sin ^{2} \Theta d \psi^{2}\right)$.

It is now manifest that for $0<r<R$, a surface of constant $r$ has ring-like topology $S^{D-2} \times S^{1}$, where the $S^{1}$ is parametrized by $\psi$. The BRs will have their event horizon at a constant value of $r<R$, and so they will inherit this topology 1 .

The ansatz and general relations.- The metric for the $d \geq 5 \mathrm{BR}$ geometry preserves most of the basic structure of (1), containing, however, additional terms that encode the gravity effects,

$$
d s^{2}=f_{1}(r, \theta)\left(d r^{2}+r^{2} d \theta^{2}\right)+f_{2}(r, \theta) d \Omega_{d-4}^{2}+f_{3}(r, \theta)(d \psi-w(r, \theta) d t)^{2}-f_{0}(r, \theta) d t^{2} .
$$

Here the range of the radial coordinate is $r_{H} \leq r<\infty$, and $r=r_{H}$ corresponds to the event horizon, where $f_{0}\left(r_{H}\right)=0$. Thus the domain of integration has a rectangular shape, and is well suited for numerical calculations.

\footnotetext{
${ }^{1}$ Moreover, for $d=5$, the $(r, \theta)$-coordinates correspond to equipotential surfaces of a scalar field sourced by a ring.
} 
The boundary conditions satisfied at $r=r_{H}$ by the other metric functions are $2 f_{1}+r_{H} \partial_{r} f_{1}=$ $\partial_{r} f_{2}=\partial_{r} f_{3}=0, w=\Omega_{H}$. As $r \rightarrow \infty$, the Minkowski spacetime background is recovered, with $f_{0}=f_{1}=1, f_{2}=r^{2} \cos ^{2} \theta, f_{3}=r^{2} \sin ^{2} \theta, w=0$. At $\theta=\pi / 2$, we impose $\partial_{\theta} f_{0}=\partial_{\theta} f_{1}=f_{2}=$ $\partial_{\theta} f_{3}=\partial_{\theta} w=0$. The boundary conditions at $\theta=0$ are $\partial_{\theta} f_{0}=\partial_{\theta} f_{1}=\partial_{\theta} f_{2}=f_{3}=\partial_{\theta} w=0$, except for the interval $r_{H}<r \leq R$, where we impose instead $f_{2}=\partial_{\theta} f_{3}=0$ on the functions $f_{2}, f_{3}$.

The metric of a spatial cross-section of the horizon is

$$
d \sigma^{2}=f_{1}\left(r_{H}, \theta\right) r_{H}^{2} d \theta^{2}+f_{2}\left(r_{H}, \theta\right) d \Omega_{d-4}^{2}+f_{3}\left(r_{H}, \theta\right) d \psi^{2} .
$$

From the above boundary conditions it is clear that the topology of the horizon is $S^{d-3} \times S^{1}$ (although the $S^{d-3}$ is not a round sphere), since $f_{3}$ is nonzero for any $r \leq R$, while $f_{2}$ vanishes at both $\theta=0$ and $\theta=\pi / 2$ (which will correspond to the poles of the $S^{d-3}$-sphere).

The radii on the horizon of the ring circle, $R_{1}$, and of the $(d-3)$-sphere, $R_{d-3}$, are unambiguously defined only for very thin rings. To obtain a measure for the deformation of the $S^{d-3}$ sphere, we compare the circumference at the equator, $L_{e}(\theta=\pi / 4$, where the sphere is fattest), with the circumference of $S^{d-3}$ along the poles, $L_{p}$,

$$
L_{e}=2 \pi \sqrt{f_{2}\left(r_{H}, \pi / 4\right)}, \quad L_{p}=2 \int_{0}^{\pi / 2} d \theta r_{H} \sqrt{f_{1}\left(r_{H}, \theta\right)},
$$

and consider, in particular, their ratio $L_{e} / L_{p}$. The radius of the $S^{1}$-circle is $\theta$-dependent. An estimate of its deformation is given by the ratio $R_{1}^{(i n)} / R_{1}^{(\text {out })}$, where $R_{1}^{(i n)}$ and $R_{1}^{(\text {out })}$ are the radii of the $S^{1}$-circle on the inside and outside of the ring, respectively,

$$
R_{1}^{(i n)}=\sqrt{f_{3}\left(r_{H}, 0\right)}, \quad R_{1}^{(o u t)}=\sqrt{f_{3}\left(r_{H}, \pi / 2\right)} .
$$

A study of the $d=5$ Emparan-Reall BR written within the Ansatz (2) can be found in Ref. [9], including the explicit form of the metric functions. Note, that the $d \geq 5 \mathrm{MP} \mathrm{BH}$ with one rotation parameter can also be written in the form (2). For BHs with a spherical horizon topology, the metric functions satisfy the same set of boundary conditions, except for $f_{2}$ and $f_{3}$ at $\theta=0$, where $\partial_{\theta} f_{2}=f_{3}=0$ for any $r>r_{H}$ (see Ref. [9] for a discussion of the $d=5$ case).

For both BRs and MP BHs, the event horizon area $A_{H}$, Hawking temperature $T_{H}$ and event horizon velocity $\Omega_{H}$ of the solutions are given by

$$
A_{H}=\left.2 \pi r_{H} V_{d-4} \int_{0}^{\pi / 2} d \theta \sqrt{f_{1} f_{2}^{d-4} f_{3}}\right|_{r=r_{H}}, \quad T_{H}=\frac{1}{2 \pi} \lim _{r \rightarrow r_{H}} \frac{1}{\left(r-r_{H}\right)} \sqrt{\frac{f_{0}}{f_{1}}}, \quad \Omega_{H}=\left.w\right|_{r=r_{H}},
$$

where $V_{d-4}$ is the area of the unit $S^{d-4}$ sphere.

The mass and angular momentum are read from the large $-r$ asymptotics of the metric functions, $g_{t t}=-f_{0}=-1+\frac{C_{t}}{r^{d-3}}+\ldots, g_{\psi t}=-f_{3} w=\sin ^{2} \theta \frac{C_{\psi}}{r^{d-3}}+\ldots$, with $(G=1)$

$$
M=\frac{(d-2) V_{d-2}}{16 \pi} C_{t}, \quad J=\frac{V_{d-2}}{8 \pi} C_{\psi} .
$$


Also, both the MP and the BR solutions satisfy the Smarr law

$$
\frac{d-3}{d-2} M=T_{H} \frac{A_{H}}{4}+\Omega_{H} J
$$

Following [5], we define a scale by fixing the mass and introduce the dimensionless 'reduced' quantities

$$
j=c_{j}^{\frac{1}{d-3}} \frac{J}{M^{\frac{d-2}{d-3}}}, \quad a_{H}=c_{a}^{\frac{1}{d-3}} \frac{A_{H}}{M^{\frac{d-2}{d-3}}}, \quad w_{H}=c_{w} \Omega_{H} M^{\frac{1}{d-3}}, \quad t_{H}=c_{t} T_{H} M^{\frac{1}{d-3}},
$$

where $c_{j}=\frac{V_{d-3}}{2^{d+1}} \frac{(d-2)^{d-2}}{(d-3)^{(d-3) / 2}}, c_{a}=\frac{V_{d-3}}{2(16 \pi)^{d-3}}(d-2)^{d-2}\left(\frac{d-4}{d-3}\right)^{\frac{d-3}{2}}, c_{w}=\sqrt{d-3}\left(\frac{(d-2)}{16} V_{d-3}\right)^{-\frac{1}{d-3}}, c_{t}=$ $4 \pi \sqrt{\frac{d-3}{d-4}}\left(\frac{d-2}{2} V_{d-3}\right)^{-\frac{1}{d-3}}$. Then both the BRs and the MP BHs are conveniently parametrized by a single dimensionless parameter which we choose to be $j$.

The numerical scheme.- We employ a numerical algorithm developed in [9, 10] which uses a Newton-Raphson method to solve for $\left(f_{i}, w\right)$, whilst ensuring that all the Einstein equations are satisfied. In this approach, the functions $f_{i}$ are expressed as products of background functions $f_{i}^{(0)}$ and unknown functions $F_{i}$. For the $f_{i}^{(0)}$ we have chosen the functions corresponding to the $d=5$ static BR (then $f_{1}^{(0)}, f_{2}^{(0)}$ and $f_{3}^{(0)}$ are essentially $V_{1}, V_{2}$ and $V_{3}$ in (2), though with some $r_{H}$-dependent corrections). The advantage of this approach is that the coordinate singularities are essentially subtracted, while imposing at the same time the event horizon topology. The reader is referred to Ref. [9] for details of this procedure.

The equations for the $F_{i}$ are solved by using a finite difference solver and, independently, by using a multi-domain spectral solver, yielding very good agreement for the results obtained by these two different numerical schemes.

In our approach, the input parameters are $R, r_{H}$ and the angular velocity $\Omega_{H}$. Although $R$ and $r_{H}$ have no invariant meaning, they provide a rough measure of the ring's $S^{1}$, and of the radius of the $S^{d-3}$ sphere, respectively, on the horizon.

In 5 dimensions, BRs exist for arbitrary values of $\Omega_{H}$, but these generic BRs possess conical singularities. Only for a critical value of the event horizon velocity they become balanced BRs [2]. Our results indicate that for $d>5$, the singularities of the unbalanced configurations are stronger. In this case, for given $R, r_{H}$ and arbitrary $\Omega_{H}$, the numerical algorithms do not converge well, except for the critical value of the event horizon velocity, where the ring is precisely balanced. This value is found by using a shooting procedure. Then the balanced solution has no singularity on and outside the horizon, possessing a finite Kretschmann scalar.

Therefore, in principle, by varying the value of $R$ (or the position of the horizon) and by adjusting the value of $\Omega_{H}$, the full spectrum of $d>5$ balanced BRs can be recovered numerically.

The results. - We have studied in a systematic way the $d=6 \mathrm{BR}$ solutions with $1.12 r_{H}<$ $R<7 r_{H}$. We have used the Smarr relation (8) to test ${ }^{2}$ the accuracy of the numerical calculations and found it very well satisfied with a typical relative error $<10^{-3}$. However, we could not obtain BRs closer to the critical point $R=r_{H}$ with high accuracy.

\footnotetext{
${ }^{2}$ We have also tested our numerical scheme by recovering the $d=5$ balanced BRs.
} 


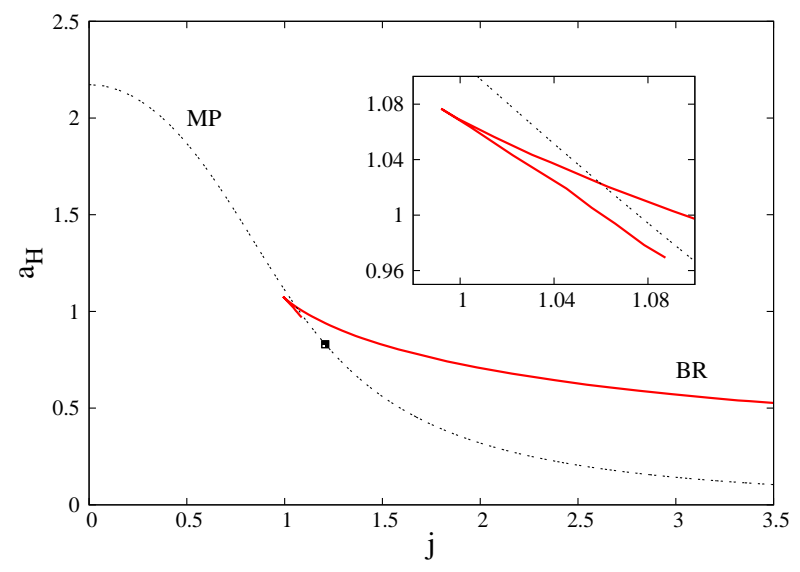

Figure 1. The reduced area $a_{H} v s$. the reduced angular momentum $j$ for $d=6$ black rings and MP black holes with a single angular momentum. The star on the MP curve indicates the critical solution where a branch of 'pinched' black holes should emerge [7, 8].

We have also constructed a number of $d=7 \mathrm{BR}$ solutions, although with much larger numerical errors. BRs should also exist for $d>7$; but so far we could not construct them within the present scheme. However, based on the results in [5], we expect that the picture found for $d=6$ is likely to hold for higher values of $d$ as well, thus being generic.

The profiles for the metric functions $f_{i}, w$ are rather similar to those of the $d=5$ balanced BR solution exhibited in Ref. 9]. The $d>5$ BRs also possess an ergo-region, defined as the domain in which the metric function $g_{t t}$ is positive. It is bounded by the event horizon and by the surface where $-f_{0}+f_{3} w=0$.

The general picture we have unveiled for $d=6$ BRs exhibits a number of remarkable similarities to the well-known $d=5$ case. We again find two branches of BR solutions whose physical differences are most clearly seen in terms of the reduced quantities $a_{H}$ and $j$ introduced above. The $a_{H}(j)$ diagram of the BRs is shown in Figure 1, with the singly rotating MP BHs included for comparison. The dependence of the reduced temperature $t_{H}$ and the reduced horizon angular velocity $w_{H}$ on the reduced angular momentum $j$ is shown in Figure 2.

The results in Figure 1 illustrate that the nonuniqueness result in 5 dimensions [2] extends also to more than 5 dimensions. We observe that the reduced area $a_{H}(j)$ has a cusp at a minimal value of $j, j_{\min }^{B R} \simeq 0.991$, where $a_{H}$ assumes its maximal value, $a_{H} \simeq 1.076$. Starting from this cusp the upper branch of solutions extends to $j \rightarrow \infty$. In the ultra-spinning regime, these correspond to thin black rings, which are well approximated by boosted black strings. The results in [5] show that a thin BR is characterized by

$$
a_{H}=2^{\frac{d-6}{(d-4)(d-3)}} \frac{1}{j^{\frac{1}{d-4}}}, \quad w_{H}=\frac{1}{2 j}, \quad t_{H}=2^{-\frac{d-6}{(d-4)(d-3)}}(d-4) j^{\frac{1}{d-4}} .
$$

We have found that this analytical result provides a good approximation for $d=6$ solutions with $j \gtrsim 2$. 

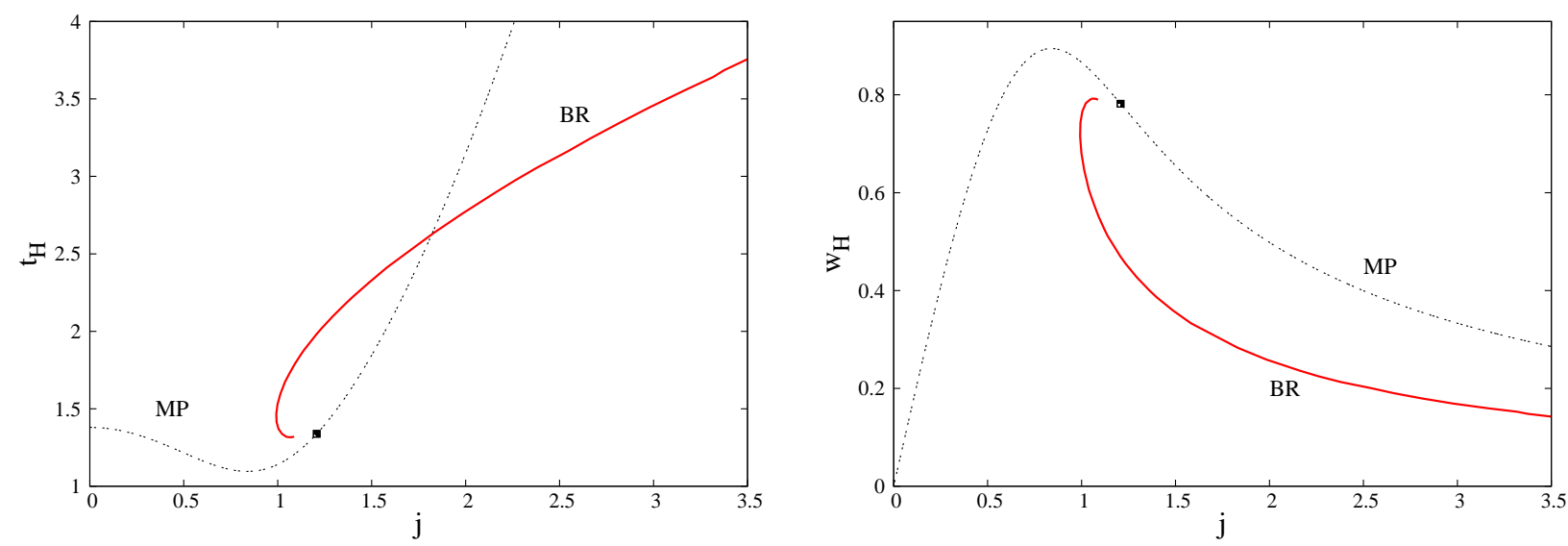

Figure 2. The reduced temperature $t_{H}$ and the reduced angular velocity $w_{H} v s$. the reduced angular momentum $j$ for $d=6$ black rings and MP black holes. The star on the MP curve indicates the critical solution where a branch of 'pinched' black holes should emerge [7, 8].

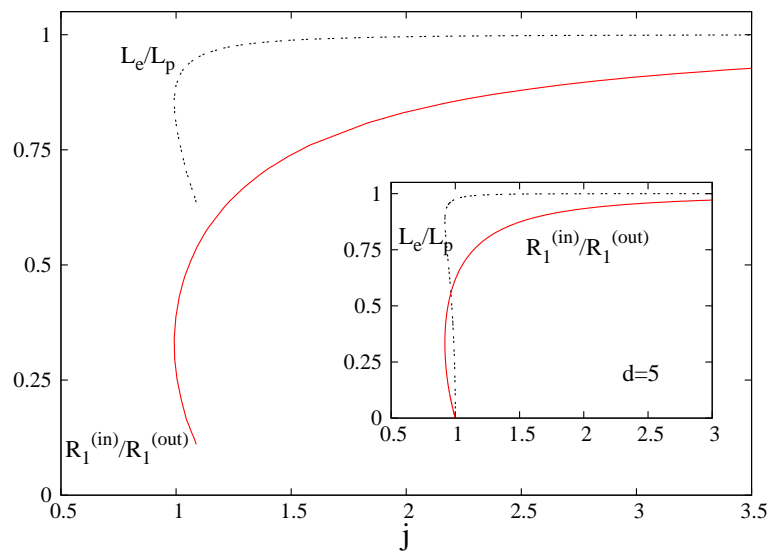

Figure 3. The ratios $L_{e} / L_{p}$ and $R_{1}^{(i n)} / R_{1}^{(o u t)}$, which encode the deformation of the horizon, $v s$. the reduced angular momentum $j$ for $d=5,6$ black ring solutions.

Starting from the cusp there is also a lower branch of BRs, the branch of fat BRs. Thus, as in 5 dimensions, in a certain range of the reduced angular momentum $j_{\min }^{B R}<j<j_{\max }$ there exist three different solutions with the same global charges. This lower branch can only have a small extent in both $j$ and $a_{H}$, since it is expected to end in a critical merger configuration [5], where a branch of pinched black holes should be approached in a horizon topology changing transition. (Extrapolations of the present data to find the location of this critical configuration indicate that it might be in the vicinity of $j_{\max } \simeq 1.15, a_{H} \simeq 0.88$ and $t_{H} \sim 1.3$.)

We conclude, that the critical merger solution has a finite, nonzero $a_{H}$ and also $t_{H}$ stays finite and nonzero. This represents a significant difference between the $d=6$ BRs and those in $d=5$ [2], where the branch of fat BRs merges with the MP BH branch in a singular solution 
with $j=1, a_{H}=0$ and $t_{H}=0$. The branch of $d=6$ 'pinched' black holes itself is expected to branch off at $j \simeq 1.27, a_{H} \simeq 0.83$ from a critical MP BH solution, along the stationary zero-mode perturbation of the GL-like instability [7, 8].

Some of these features can be seen in Figure 3, where we show the deformations of the $S^{1}$ and $S^{3}$-components of the horizon, as defined by (4) and (5) as functions of the reduced angular momentum. As the horizon topology changing solution is approached, $L_{e}, L_{p}$ and $R_{1}^{(o u t)}$ stay

finite and nonzero, while $R_{1}^{(i n)} \rightarrow 0$. In contrast, for $d=5$, when the hole inside the ring shrinks to zero size the outer radius goes to infinity as the singular solution is approached [11]. (The inset in Figure 3 demonstrates the $d=5$ case.)

Further remarks.- In this work, by using a special coordinate system, we have been able to formulate the problem of $d>5$ balanced BRs in a numerically manageable manner, and to find such BR solutions in a nonperturbative way. Our results for $d=6$ confirm the conjecture of [5] for the phase diagram of single black objects. Perhaps our most interesting new result is the occurrence of a cusp in the $a_{H}(j)$ BR diagram with a small branch of fat BRs.

For reasons of simplicity, we have restricted our calculations to vacuum BR solutions with a single angular momentum. But our methods should readily extend to more general situations (e.g. to the presence of matter fields). Moreover, it should be interesting to consider multiblack hole configurations and to extend the phase diagram proposed in [12 to the fully nonperturbative regime. However, it currently remains a numerical challenge to connect the BR and MP branches of $d>5$ spinning solutions by constructing the corresponding set of 'pinched' black holes.

Acknowledgements.- We would like to thank N. A. Obers for helpful discussions. We gratefully acknowledge support by the DFG, in particular, also within the DFG Research Training Group 1620 "Models of Gravity".

\section{References}

[1] R. C. Myers and M. J. Perry, Annals Phys. 172, 304 (1986).

[2] R. Emparan and H. S. Reall, Phys. Rev. Lett. 88 (2002) 101101 [arXiv:hep-th/0110260].

[3] R. Emparan and H. S. Reall, Class. Quant. Grav. 23 (2006) R169 arXiv:hep-th/0608012.

[4] R. Emparan and H. S. Reall, Living Rev. Rel. 11 (2008) 6 [arXiv:0801.3471 [hep-th]].

[5] R. Emparan, T. Harmark, V. Niarchos, N. A. Obers and M. J. Rodriguez, JHEP 0710 (2007) 110 arXiv:0708.2181 [hep-th]].

[6] R. Emparan, T. Harmark, V. Niarchos and N. A. Obers, JHEP 1004, 046 (2010) arXiv:0912.2352 [hep-th]];

R. Emparan, T. Harmark, V. Niarchos and N. A. Obers, Phys. Rev. Lett. 102 (2009) 191301 arXiv:0902.0427 [hep-th]].

[7] O. J. C. Dias, P. Figueras, R. Monteiro, J. E. Santos and R. Emparan, Phys. Rev. D 80 (2009) 111701 arXiv:0907.2248 [hep-th]]. 
[8] O. J. C. Dias, P. Figueras, R. Monteiro and J. E. Santos, Phys. Rev. D 82, 104025 (2010) arXiv:1006.1904 [hep-th]].

[9] B. Kleihaus, J. Kunz, E. Radu and M. J. Rodriguez, JHEP 1102, 058 (2011) arXiv:1010.2898 [gr-qc]].

[10] B. Kleihaus, J. Kunz, E. Radu, Phys. Lett. B678, 301-307 (2009). arXiv:0904.2723 [hep-th]].

[11] H. Elvang, R. Emparan and A. Virmani, JHEP 0612, 074 (2006) hep-th/0608076.

[12] R. Emparan and P. Figueras, JHEP 1011 (2010) 022 [arXiv:1008.3243 [hep-th]]. 